\title{
Ultrastructure of articular cartilage in experimental immobilization
}

\section{S. ROY}

Department of Pathology, All-India Institute of Medical Sciences, New Delhi-16, India

The effect of immobilization on the articular cartilage by light microscopy has been studied by many workers (Salter and Field, 1960; Evans, Eggers, Butler, and Blumel, 1960; Trias, 1961; Hall, 1963; Crelin and Southwick, 1964; Roy, 1970). Varying degrees of degeneration and necrosis, occurring mostly in the areas that come in contact with the opposing cartilage, have been observed. The fine changes occurring in the early stages of immobilization can be appreciated only by ultramicroscopical examination, which also helps to determine changes in the cell organelles in the later stages. The effects of immobilization in the areas that remain free from contact with the opposing cartilage are also better appreciated by electron microscopy.

\section{Material and methods}

A series of 22 1-year-old male rabbits was used in this experiment. The right knee joint of each animal was immobilized in plaster of Paris in a position midway between flexion and extension. The hip and ankle joints on the same side were also included in the plaster to ensure full immobilization of the knee joint. The left knees served as controls.

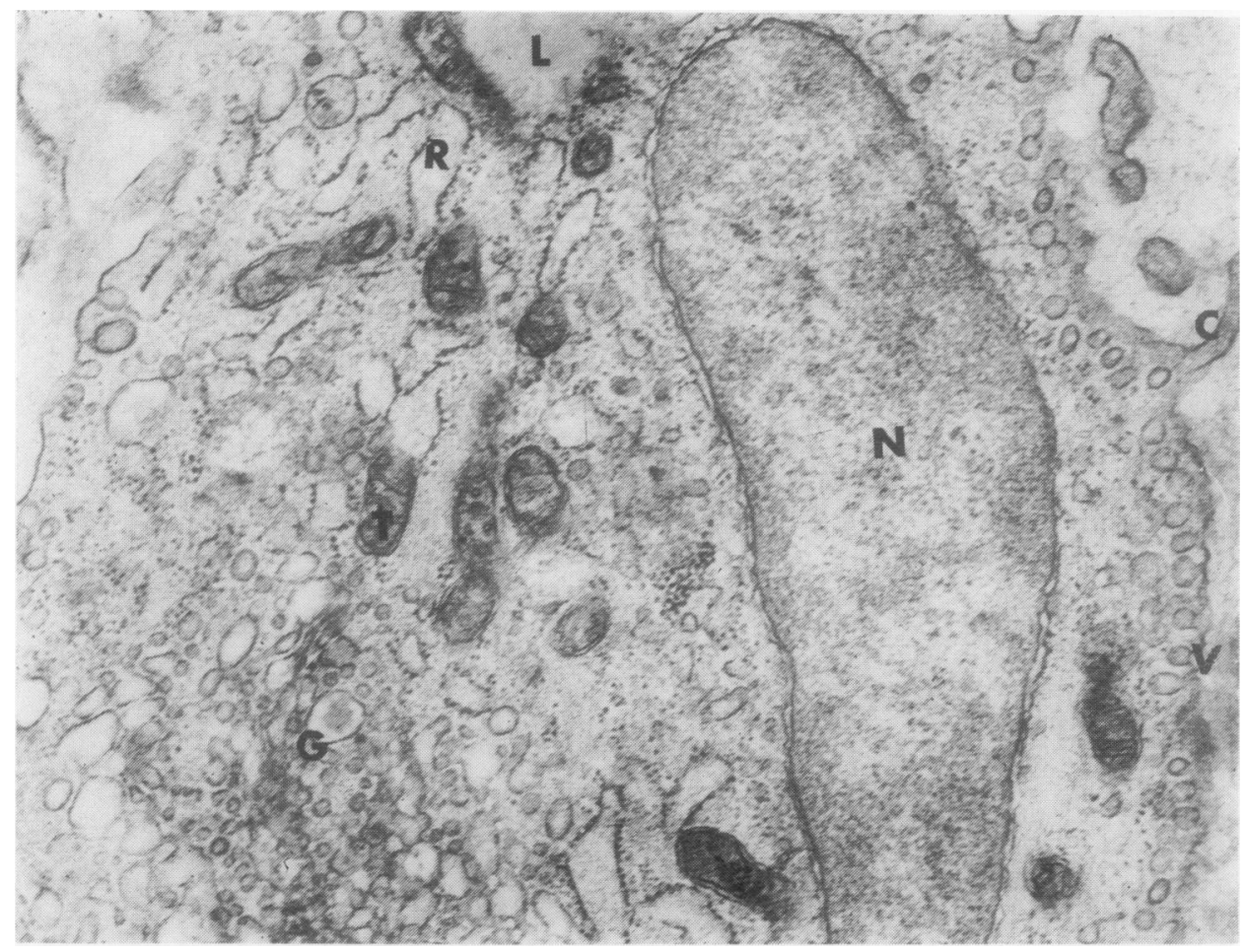

FIG. 1 Normal chondrocyte, showing cytoplasmic processes $(C)$, micropinocytotic vesicles $(V)$, rough endoplasmic reticulum $(R)$, mitochondria $(T)$, Golgi apparatus $(G)$, lipid droplet $(L)$, and nucleus $(N) . \times 32,000$. 
Two or three animals were killed after $3,5,10,21,42$, 63,84 , and 168 days of immobilization. Before they were killed, the animals were anaesthetized with ether and, after removal of the plaster from the right side, both knee joints were opened and small pieces of articular cartilage were collected from the medial femoral condyles of both joints. On the test side, pieces were taken from the areas of contact between the tibial and femoral condyles and also from areas free from contact with the opposing cartilage. The specimens were immediately put into 1 per cent. cold osmium tetroxide in veronal acetate buffer (Palade, 1952) and, after fixation for 2 hours, the tissue was processed and examined by the methods already described (Meachim and Roy, 1967).

\section{Results}

\section{CONTROLS}

Cartilage from the left femoral condyle (control joint) showed normal ultrastructural appearances with zonal distribution of chondrocytes (Davies, Barnett, Cochrane, and Palfrey, 1962; Palfrey and Davies, 1966; Ghadially and Roy, 1969). A normal cell in the middle zone showing characteristic ultrastructural features is shown in Fig. 1.

\section{IMMOBILIZED JOINTS}

\section{Non-contact (free) areas}

In joints immobilized for 3 or 5 days, chondrocytes from areas of cartilage that were not in contact with each other during immobilization (free areas) showed dilatation of the cisternae of rough endoplasmic reticulum (RER) and a prominent Golgi apparatus (Fig. 2) in many chondrocyles of the middle zone. The matrix appeared normal.

Degenerative changes could be detected in a few chondrocytes from joints immobilized for 10 days.

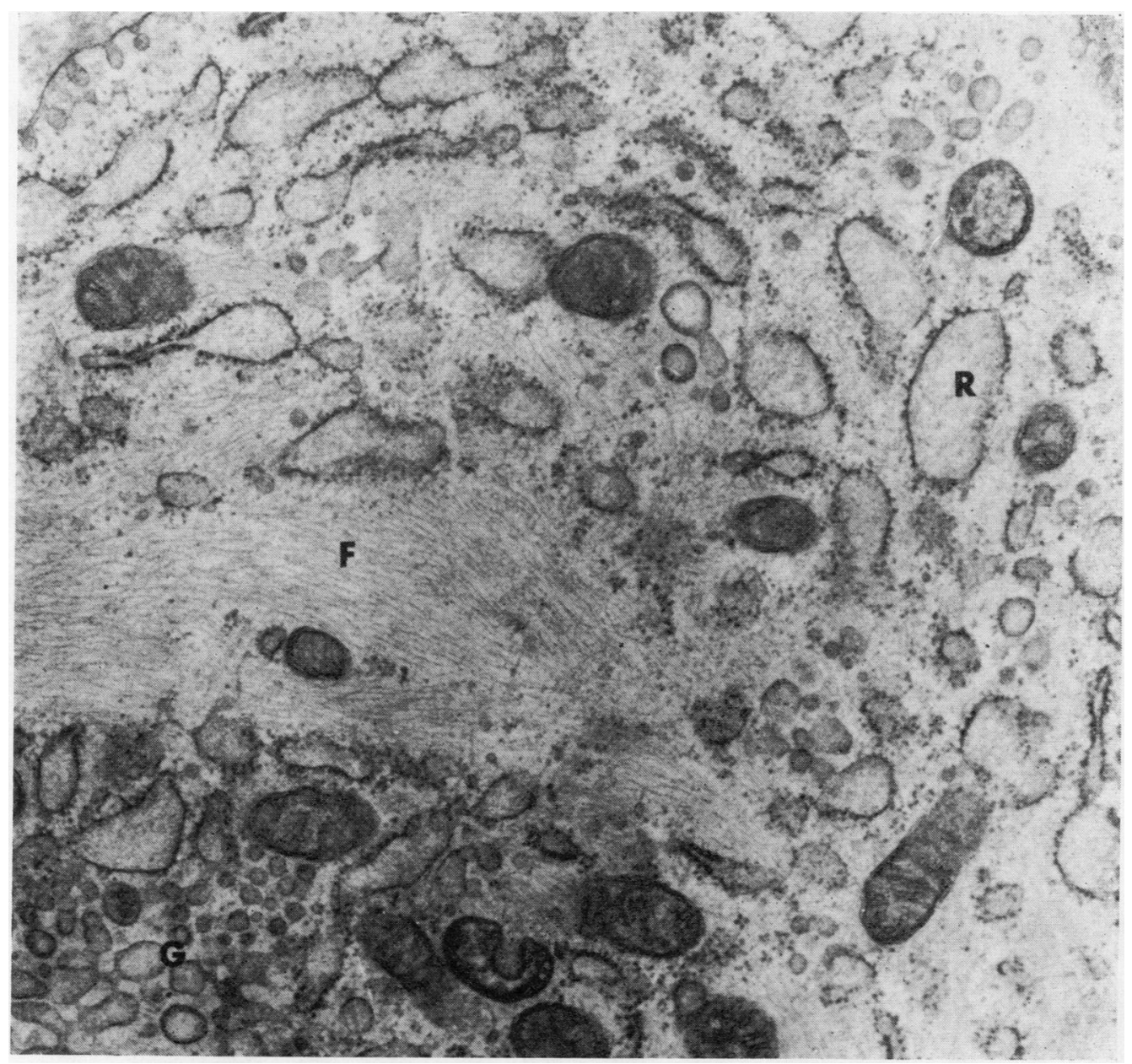

FIG. 2 Chondrocyte, showing slight dilatation of some of the rough endoplasmic reticulum cisternae (R), a few fine filamentous fibres $(F)$, and Golgi apparatus $(G)$ after 5 days' immobilization. $\times 33,000$. 


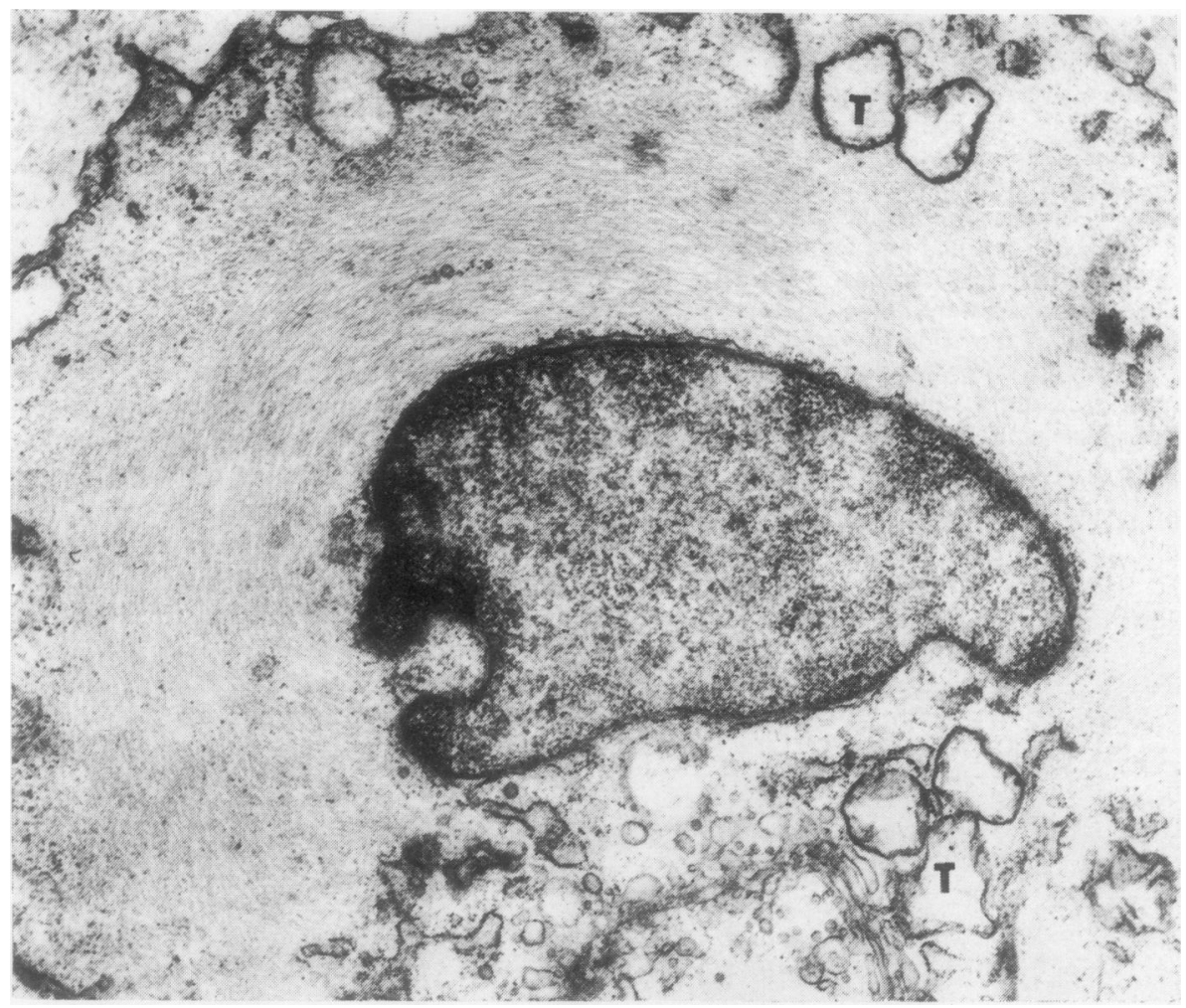

FIG. 3 Chondrocyte, showing mitochondria $(T)$ with almost complete absence of cristae, and more filamentous fibres after 10 days' immobilization. $\times 16,000$.

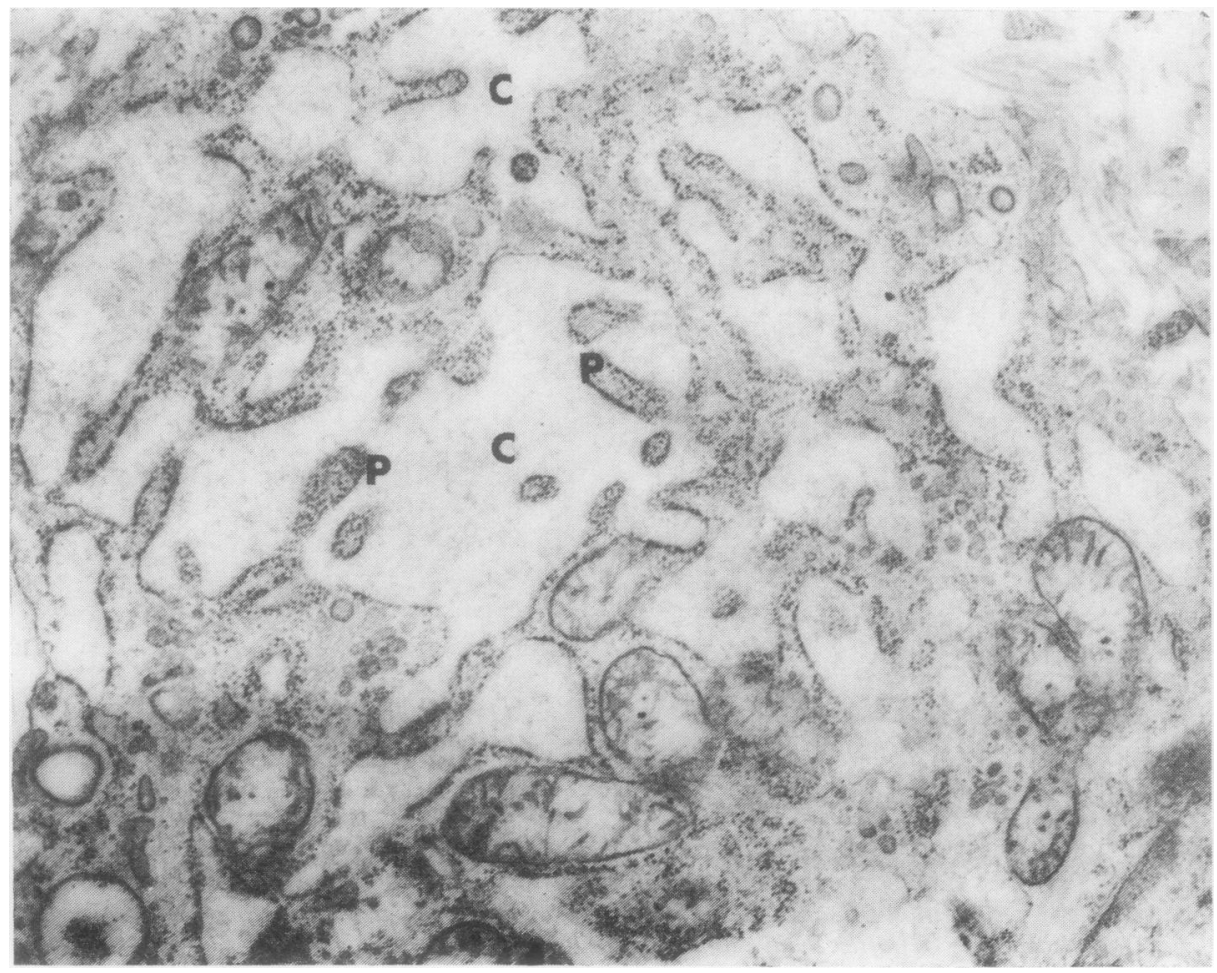

FIG.4 Chondrocytes, showing markeddilatation of cisternae (C) of rough endoplasmicreticulum with many projections $(P)$ of walls of endoplasmic reticulum within them, after 63 days' immobilization. $\times 26,500$. 
These were manifested by swelling of the mitochondria with reduction or complete loss of the cristae (Fig. 3), slight to moderate dilatation of the cisternae of the RER, and increased numbers of cytolysomes. These changes were seen mostly in the superficial and middle zones. In addition, a few cells in the middle zone contained moderate to large amounts of fine filamentous fibres replacing other organelles from the cell cytoplasm.

The degenerative changes were observed in increasing degrees of severity in the later samples, when marked dilatation of RER cisternae with severe disorganization (Fig. 4) of the RER system and swelling of the mitochondria were frequently noted. There was also a further increase in fine filamentous fibres (Fig. 5) in many chondrocytes; these occupied most of the cell cytoplasm, the remaining organelles being scattered at the periphery of the cell. This last feature was particularly marked after 63,84 , and 168 days, when fine filamentous fibres could often be seen even in the chondrocytes of the superficial zone where these fibres rarely occur (Palfrey and Davies, 1966; Ghadially and Roy, 1969). Some chondrocytes of the superficial zone lost their ovoid or elongated shape and became rounded.

Large numbers of lipid droplets were also seen in many chondrocytes in both the superficial and middle zones. An increase in lipid droplets was observed in chondrocytes after 6 weeks' immobilization, but was much more pronounced in the later stages. Many cells showed many lipid droplets occupying most of the cytoplasm (Fig. 6, overleaf). The lipid droplets were much more numerous than in normal rabbits (Ghadially and Roy, 1969) or in the control specimens, and most of them were larger than those seen in normal chondrocytes.

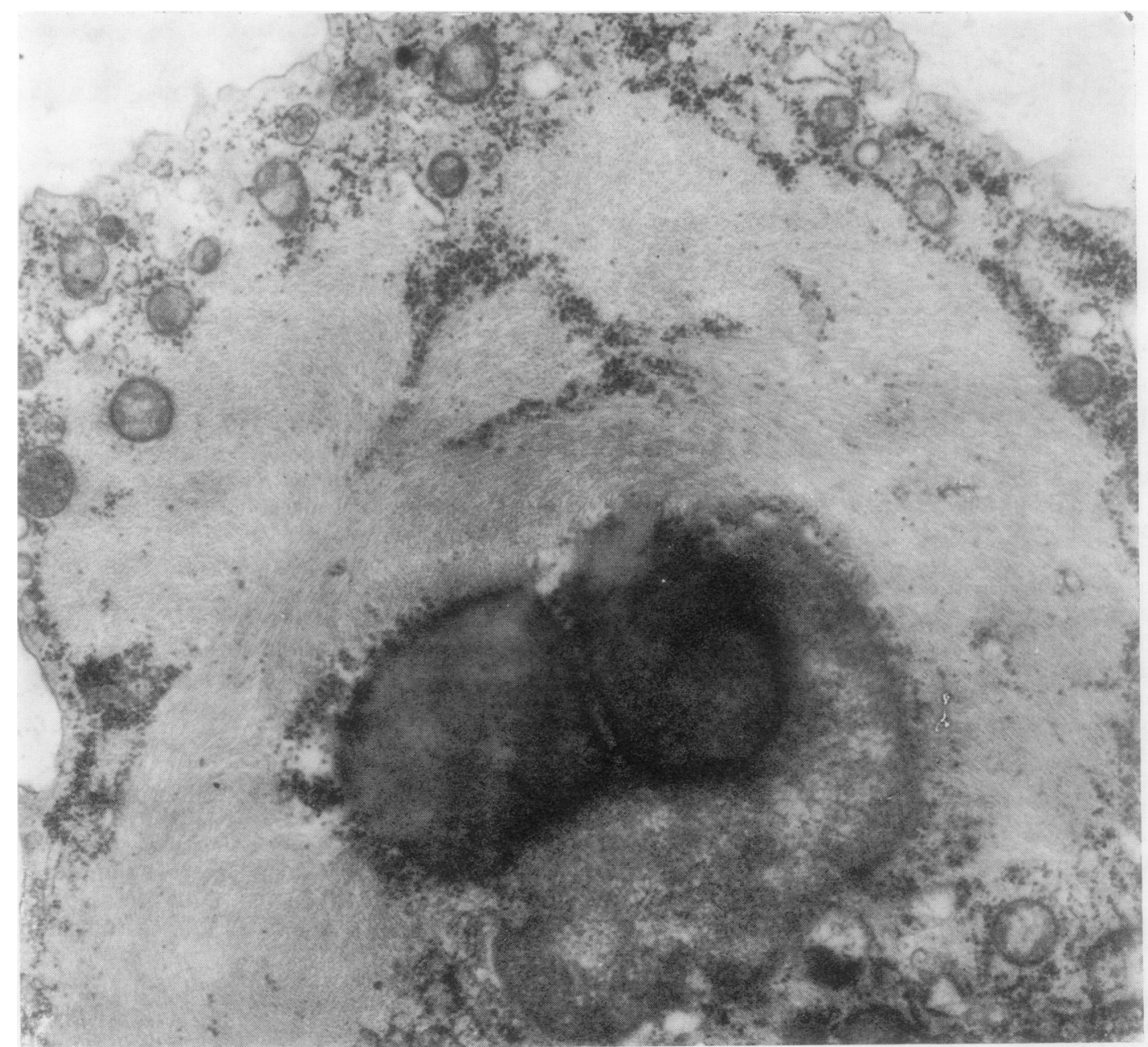

FIG. 5 Chondrocyte, showing much fine filamentous material replacing cytoplasmic organelles and two lipid droplets, after 42 days' immobilization. $\times 26,500$. 


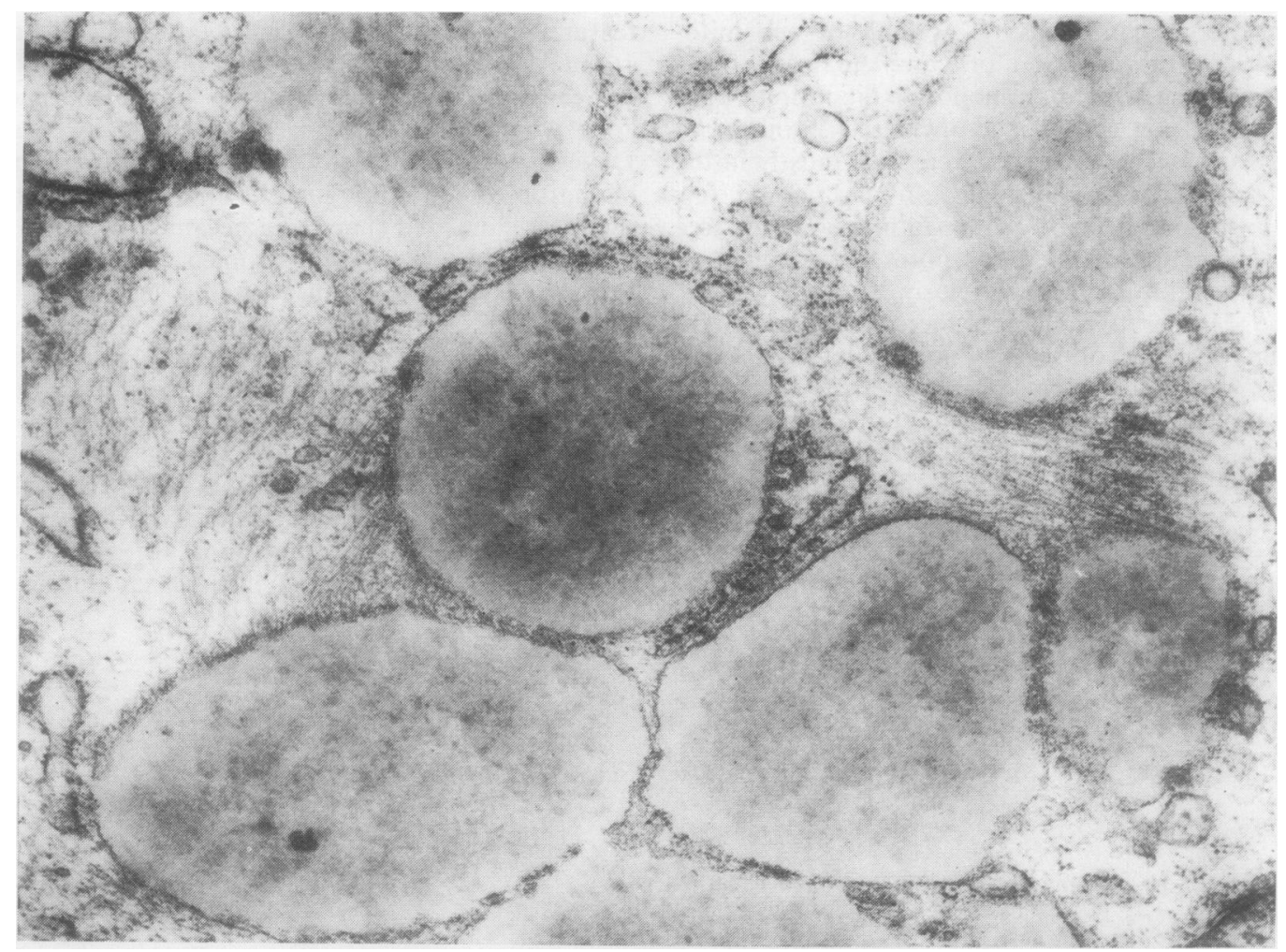

FIG. 6 Chondrocyte containing many lipid droplets after 168 days' immobilization. $\times$ 32,000.

Although most of these lipid droplets were of medium density, a few cells contained a few very pale electronlucent lipid droplets.

A significant increase in the number of lysosomal bodies was noted in samples from 63 days onwards. These were of various sizes, usually bounded by a single or double membrane, and they contained electron dense whorled or homogeneous structures (Fig. 7). These appeared to have arisen from degenerated cytoplasmic organelles and were thus considered to be cytolysomes. Occasional lysosomal bodies were lined by a double membrane and showed very small structures at the periphery resembling attenuated cristae within them (Fig. 7), which was suggestive of their origin from degenerated mitochondria. Similar structures have been reported in human chondrocytes from osteoarthritic cartilage (Roy and Meachim, 1968).

Necrosis of chondrocytes was also evident in specimens examined after 63,84 , and 168 days, in which scattered dying or necrosed cells or cell fragments could often be seen (Fig. 8). This feature was particularly marked in the superficial zone after 84 and 168 days of immobilization. It should, however, be pointed out that, even at this stage, many chondrocytes showed normal ultrastructural appearances, especially in the middle and deeper zones.

The matrix appeared normal in most of the samples, especially in the middle and deeper zones. However, in the superficial zone in the late stages of immobilization (63 to 168 days), the matrix appeared loose and granular with fragmentation of collagen, and the middle zone also showed focal areas where the matrix appeared loose and the collagen occurred in short lengths.

\section{Contact areas}

Here many chondrocytes appeared normal at the early stages (3rd and 5th days), showing a welldeveloped Golgi apparatus and a slight to moderate dilatation of the cisternae of RER, an appearance suggestive of increased activity of the cells. However, a few cells in the superficial zone showed degenerative changes, as described above. In subsequent specimens degenerative change and necrosis of the chondrocytes, similar to those observed in the contact-free areas, became increasingly severe and were much more pronounced. Thus degeneration was already well marked in 21-day specimens, when many cells 


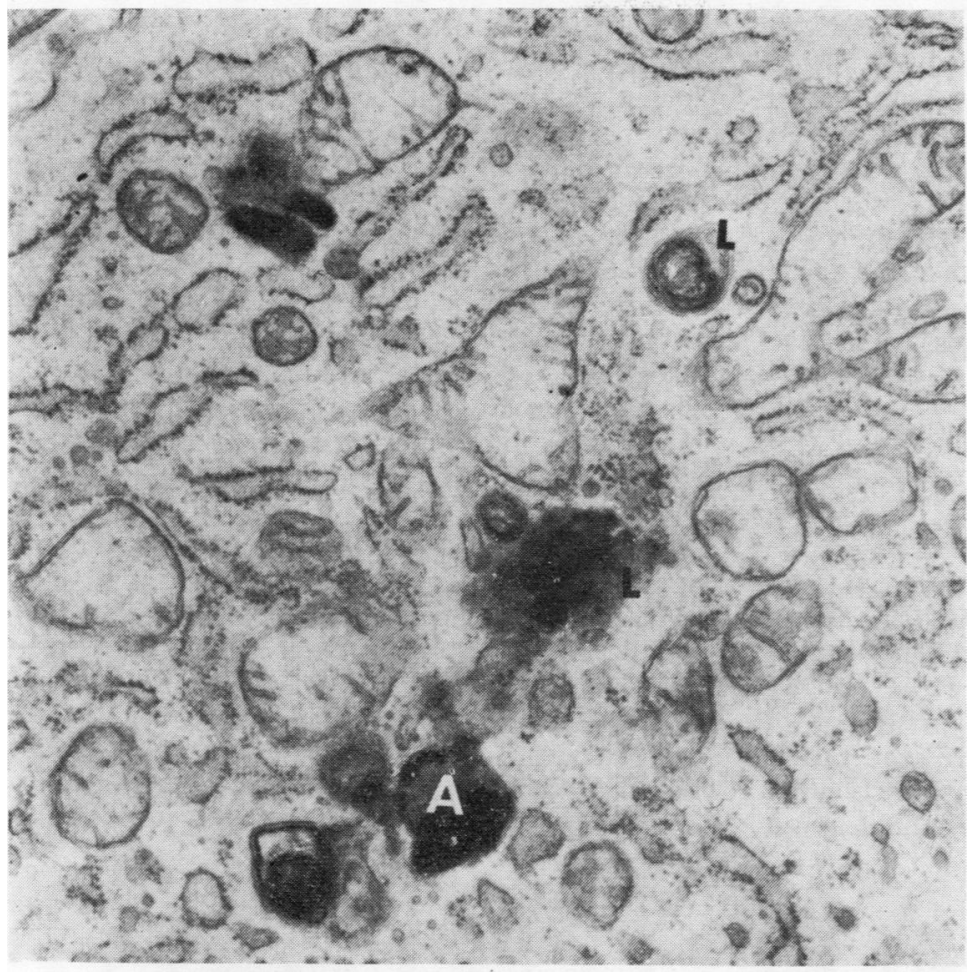

FIG. 7 Chondrocyte containing many lysosomal bodies $(L)$, one of which $(A)$ shows a double membrane and small cristae-like structures inside. In other mitochondria there is shortening or loss of cristae after 63 days' immobilization. $\times 32,000$.

FIG. 8 Superficial zone, showing a necrosed chondrocyte after 84 days' immobilization. $\times 31,000$.

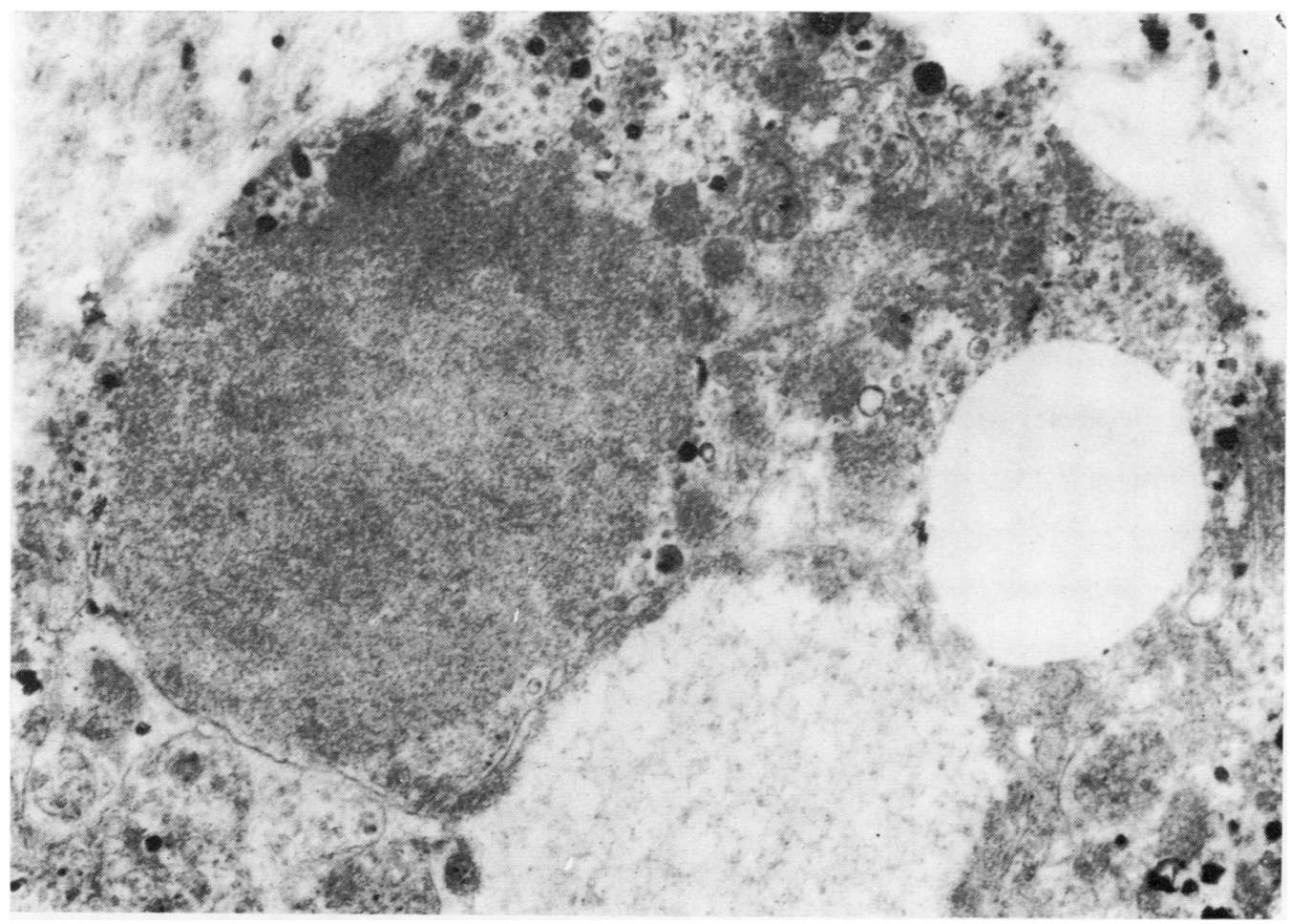


showed severe degeneration or necrosis, specially in the superficial zone. In the later stages, the surface of the cartilage was very irregular with actual fibrillation, and many dead or degenerated cells or cell fragments could be seen, especially in the superficial zone (Figs 9 and 10). The matrix was very loose, especially in the superficial zone, with irregularly orientated and fragmented collagen fibres. Gross fibrillation with degeneration and necrosis of a large part of the cartilage was seen in 168-day specimens.

\section{Discussion}

The present study demonstrates that degeneration and necrosis of the chondrocytes occur in the cartilage not only in areas of compression in immobilized joints but also in the contact-free areas. The changes in the contact areas, however, appear earlier and are much more severe, resulting in the early onset of necrosis (Roy, 1970). The degenerative changes observed in the present study (swelling of mitochondria with loss of cristae, marked dilatation of the cisternae of the RER with disorganization of the RER system, accumulation of excessive amount of fine filamentous fibres, lipid, and lysosomal bodies) are somewhat similar to those that occur in the cartilage in other pathological conditions; for example, in experimental haemarthrosis in the rabbit (Roy, 1968) or in human osteoarthritis (Meachim and Roy, 1967; Roy and Meachim, 1969). Degeneration and necrosis of a few cells may be seen even in normal rabbit cartilage (Palfrey and Davies, 1966; Ghadially and Roy, 1969) but they usually lie in the deeper zone. In the present experiment, the changes were much more frequent, and were present in many chondrocytes, mostly in the superficial and middle zones.

Degeneration and necrosis of early onset and severe degree in the contact area during immobilization have already been reported after examination with the light microscope (Salter and Field, 1960; Trias, 1961; Roy, 1970), and these are substantiated in the present study. The changes appear to be largely due to the effect of mechanical compression, which will not only produce damage of the opposing surfaces due to pressure but will also prevent the

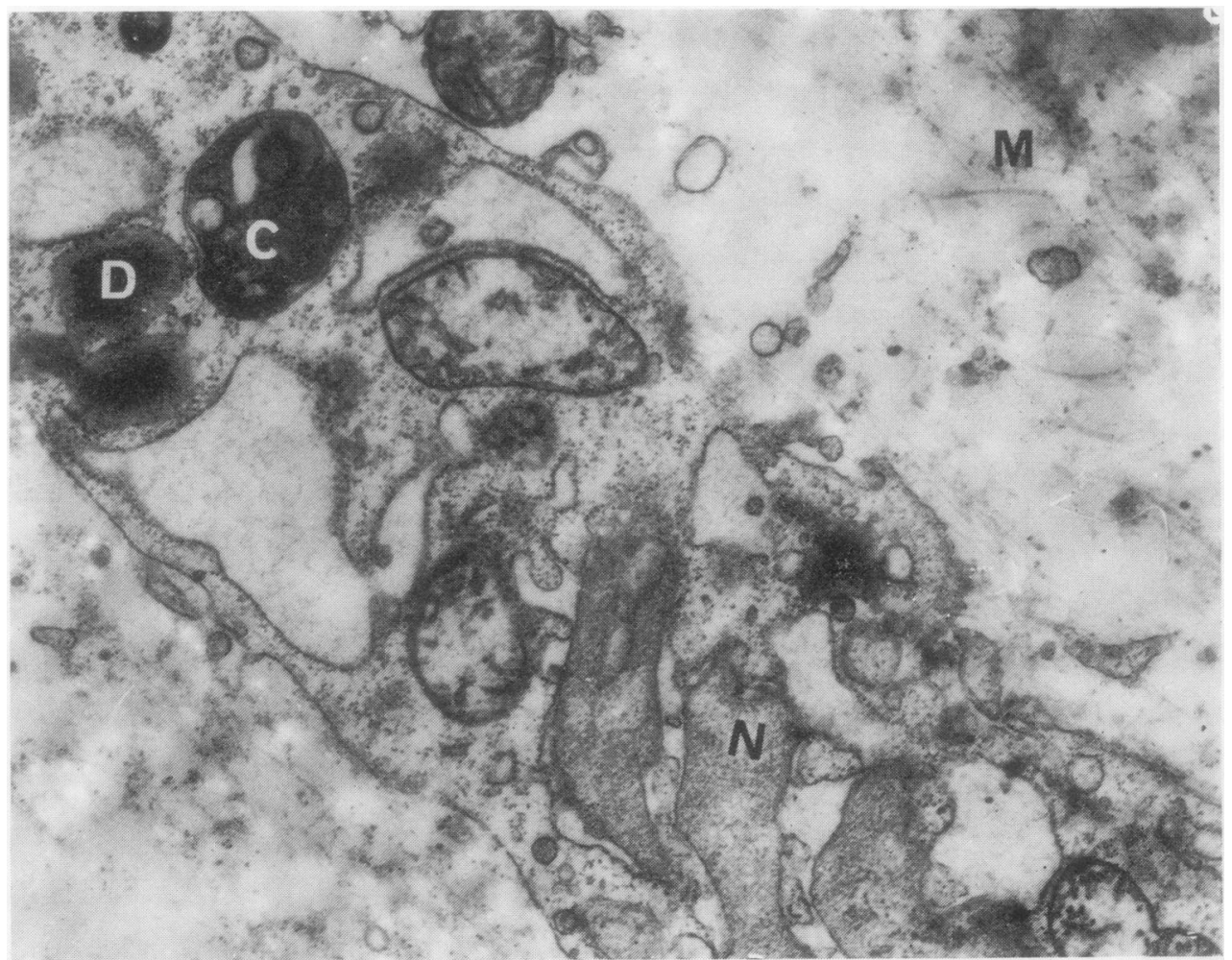

FIG. 9 Superficial zone with a chondrocyte, showing dilatation of cisternae of rough endoplasmic reticulum, swelling of mitochondria, cytolysomes $(C)$, lipid $(D)$, and segmented nucleus $(N)$. Note looseness of the matrix $(M)$ in which are seen scattered cytoplasmic structures, probably of a disintegrated cell, after 168 days' immobilization. $\times 26,000$. 


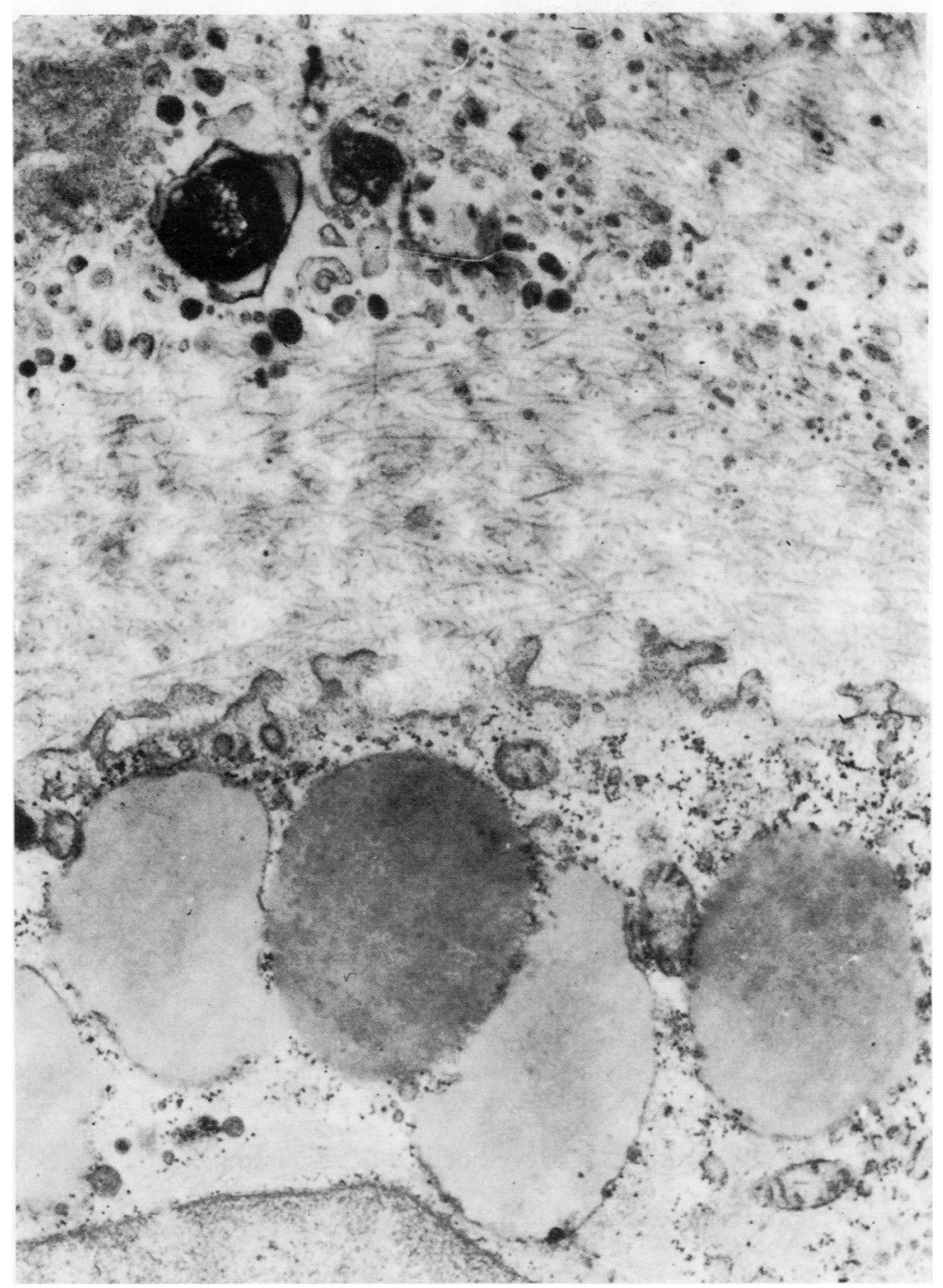

FIG. 10 Middle zone with a chondrocyte containing many lipid droplets and remnants of a disintegrated chondrocyte, after 63 days' immobilization. $\times 32,000$.

diffusion of nutrient synovial fluid into the compressed cartilage. Immobilization may also be a contributory factor in hindering the entry of synovial fluid into the articular cartilage through the absence of the pumping action of joint movement which is believed to aid its diffusion (Ekholm, 1955). Thus the changes in the contact areas seem to represent a combined effect of compression and immobilization.

The effects of immobilization alone (without any mechanical compression) on articular cartilage should be observed in areas free from contact with the opposing cartilage during immobilization. These areas also show degeneration and necrosis, though of slower onset and of less severity than in the contact areas. However, the exact mechanism of the production of these degenerative changes in chondrocytes which are due purely to the immobilization of a joint is not clear. The nutrition of the articular cartilage of adult rabbit, as used in this experiment, is believed to be derived entirely from the synovial fluid (Hodge and McKibbin, 1969). It is possible that degeneration in contact free areas is due to the limitation of joint movement which reduces the diffusion of synovial fluid into the articular cartilage. Another possibility is that the changes are due to the reduced production of synovial fluid which may result from alterations in the synovial membrane during immobilization. The synovial cells do show various degenerative changes on ultrastructural examination even in the early stages of immobilization and the synovial membrane as a whole shows marked atrophy, apparent on both light and electron microscopical examination, in the late stages (personal observation). Degeneration and atrophy of synovial membrane are likely to result in diminished production of synovial fluid, and to reduce the 
supply of cartilage nutrition. It appears likely that the degeneration seen in contact free areas may be due to atrophy of the synovial membrane leading to defective production of synovial fluid. This atrophy is probably the same kind of disuse atrophy as that which occurs in the periarticular tissues accompanied by reduction of synthesis of mucopolysaccharides and collagen in immobilized joints (Akeson, 1966; Akeson, Amiel, and LaViolette, 1967). Similar changes have also been observed in atrophic limbs following neurectomy (Brooke and Slack, 1969). It is also possible that the degeneration is partly due to the absence of the pumping action of joint movement which inhibits the diffusion of the available synovial fluid into the cartilage.

The fact that the degenerative changes appear mostly in the superficial and middle zones suggests that only these areas of articular cartilage receive nutrition from the synovial fluid, while the deeper zone is at least partly supplied by the subchondral blood vessels by a process of diffusion. This idea is supported by a recent study of Greenwald and Haynes (1969), who demonstrated the presence of a nutritional pathway from the medullary cavity to the articular cartilage of the femoral head in both man and rabbit.

\section{Summary}

The ultrastructure of articular cartilage from the knee joints immobilized for from 3 to 168 days was studied in 22 rabbits with particular reference to the changes occurring in areas that remained free from contact with the surface of the opposing cartilage at the time of immobilization. Degenerative changes in chondrocytes of the superficial and middle zones (manifested by dilatation of the cisternae of rough endoplasmic reticulum, swelling of the mitochondria with loss of cristae and the accumulation of fine filamentous fibres, many lipid droplets, and lysosomal bodies) appeared after 10 days of immobilization and became increasingly severe in later samples. This was ultimately followed by necrosis of many chondrocytes of the superficial and middle zones, accompanied by loosening of matrix and fragmentation of collagen, predominantly in the superficial zones. The degenerative changes and necrosis appeared earlier and were much more pronounced in specimens of cartilage from areas that were in contact with the opposing cartilage at the time of immobilization. The possible mechanism of cartilage changes in these two sites has been discussed.

\section{References}

AKeson, W. H. (1966) J. Bone Jt Surg., 48-A, 808 (The connective tissue response to immobility).

A Amiel, D., AND LaViolette, D. (1967) Clin. Orthop., 51, 183 (The connective tissue response to immobility).

Brooke, J. W., AND SlaCK, H. G. B. (1959) Ann. rheum. Dis., 18, 129 (Metabolism of connective tissue in limb atrophy in the rabbit).

Crelin, E. S., AND SouthWick, W. O. (1964) Anat. Rec., 149, 113 (Changes induced by sustained pressure in the knee joint articular cartilage of adult rabbits).

Davies, D. V., Barnett, C. H., Cochrane, W., AND Palfrey, A. J. (1962) Ann. rheum. Dis., 21, 11 (Electron microscopy of articular cartilage in the young adult rabbit).

EкноLм, R. (1955) Acta Anat. (Basel), 24, 329 (Nutrition of articular cartilage. A radioautographic study).

Evans, E. B., EgGers, G. W. N., Butler, J. K., AND Blumel, J. (1960) J. Bone Jt Surg., 42-A, 737 (Experimental immobilization and remobilization of rat knee joints).

Ghadially, F. N., AND RoY, S. (1969) 'Ultrastructure of Synovial Joints in Health and Disease', pp. 33-59. Butterworths, London.

Greenwald, A. B., AND Haynes, D. W. (1969) J. Bone Jt Surg., 51-B, 747 (A pathway for nutrients from the medullary cavity to the articular cartilage of the human femoral head).

HaLl, M. C. (1963) Ibid., 45-A, 36 (Cartilage changes after experimental immobilization of the knee joint of the young rat).

HodGE, J. A., AND MCKIBBIN, B. (1969) Ibid., 51-B, 140 (The nutrition of mature and immature cartilage in rabbits).

MeAChIM, G., AND RoY, S. (1967) Ann. rheum. Dis., 26, 50 (Intracytoplasmic filaments in the cells of adult human articular cartilage).

Palade, G. E. (1952) J. exp. Med., 95, 285 (A study of fixation for the electron microscopy).

Palfrey, A. J., AND DAVIeS, D. V. (1966) J. Anat. (Lond.), 100, 213 (The fine structure of chondrocytes).

Roy, S. (1968) Arch. Path., 86, 69 (Ultrastructure of articular cartilage in experimental haemarthrosis).

(1970) Ind. J. Orthop. (In press) (Effects of immobilisation on articular cartilage).

- AND MeACHIM, G. (1968) Ann. rheum. Dis., 27, 544 (Chondrocyte ultrastructure in adult human articular cartilage).

SALTER, R. B., AND FIELD, P. (1960) J. Bone Jt Surg., 42-A, 31 (The effect of continuous compression on living articular cartilage. An experimental investigation).

TriAS, A. (1961) Ibid., 43-B, 376 (Effect of persistent pressure on the articular cartilage. An experimental study). 